In biology or in circuits, this would be done by placing the system in state $\mu^{\text {new }}$-for example, driven by external inputsand enabling a learning process that allows all $T_{\mathrm{ij}}$ to increment. The information needed by each synapse is local-the increment for synapse ij does not depend on the global structure of the new state or past memories, but only on $\mu_{i}^{\text {new }}$ and $\mu_{j}^{\text {new }}$.

Under this algorithm, when random starting states are chosen, some stored memories are much more accessible than others, that is, considerably larger numbers of randomly chosen initial states lead to some memories than to others. This is a vagary of the particular set of memories which have been learned. It occurred to us that it would be possible to reduce this unevenness of access (which can be intuitively described as the " $50 \%$ of all stimuli remind me of sex" problem) by 'unlearning'.

Specific unlearning was implemented by choosing starting states at random; when a final equilibrium state $\mu^{\mathrm{f}}$ was reached it was weakly unlearned by the incremental change

$$
\text { unlearn } \mu^{\mathrm{f}} \Delta T_{\mathrm{ij}}=-\varepsilon \mu_{\mathrm{i}}^{\mathrm{f}} \mu_{\mathrm{j}}^{\mathrm{f}}, 0<\varepsilon \ll 1
$$

Figure 1 illustrates the effect of unlearning on the accessibility of five stored memories in a set of 32 neurones. Accessibility is quantitatively defined as the fraction of random initial states leading to a particular final stable state or group of states. The unevenness of the lines is due in part to statistical noise in the simulation. The accessibility of the nominal assigned memories initially ranges over a factor of 3 , but converges with unlearning to a spread of only a factor of 1.4 . Thus the accessibility is much more uniform (or in Crick-Mitchison terms, the relative stability of the modes made more uniform) after specific unlearning, and the system will have functionally improved recall.

In our model the storage of a set of assigned memories in $T_{\mathrm{ij}}$ also produces a set of spurious stable states which were not inserted as memory states. One of the strong effects of unremembering is to reduce the total accessibility of spurious states, as shown by the solid line in Fig. 1.

The qualitative reason for the success of unlearning comes from the behaviour of the 'energy' $E$, defined for any state $\mu$ as

$$
E=-\sum_{\mathrm{i} \neq \mathrm{j}} \sum_{\mathrm{j}} T_{\mathrm{ij}} \mu_{\mathrm{i}} \mu_{\mathrm{j}}
$$

The change of neural state with time according to the asynchronous algorithm monotonically decreases $E$ until a final stable state is reached-either a stored memory or a spurious memory. Any stable state $\mu^{\mathrm{m}}$ has, for a given $T_{\mathrm{ij}}$, an energy $E^{m}$. There is a strong tendency for the states having the deepest energy valleys to collect from the largest number of random starting states, that is, deep valleys are also broad. When a final state $\mu^{\mathrm{f}}$ is unlearned, its energy $E^{\mathrm{f}}$ is specifically raised and its valley of collection diminished relative to other states. While this argument indicates why accessibility of stored memories should be made more nearly even by unlearning, only a detailed analysis shows why the spurious states should be so sensitive to it. Too much unlearning will ultimately destroy the stored memories.

We have identified a class of spurious states, which in their most elementary form have their origin in triples. As an example on 16 neurones
Memory 1
Memory 2
Memory 3
Spurious memory

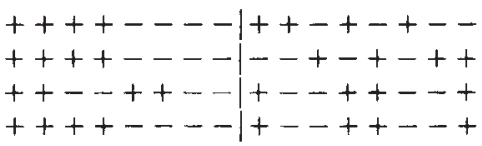

The stability of the spurious memory is enhanced if the first half of memory 3 is weakly correlated with memories 1 and 2 . Mathematical analysis of the statistical stability of such spurious states shows that they are typically less stable than the assigned memories, and that the stability will also depend on correlations with other memories. The nature of these spurious states can be described by analogy in terms of higher level function by the example

$\begin{array}{ll}\text { Memory } 1 & \text { Walter, white } \\ \text { Memory } 2 & \text { Walter, black } \\ \text { Memory } 3 & \text { Harold, grey } \\ \text { Spurious memory } & \text { Walter, grey }\end{array}$

where grey is taken as a category equally resembling black and white. This spurious state is more stable when 'Harold' and 'Walter' have a significant correlation-perhaps 'Harold' and 'Harry'. These particular spurious states are not simply transitive logical associations of the form $A \leftrightarrow B, B \leftrightarrow C ; \rightarrow A \leftrightarrow C$. They are truly spurious 'illogical' associations, but perhaps 'plausible' as they come from correlations in the structure of memories.

In our simple system, unlearning improves memory function both by the equalization of accessibility and the suppression of spurious memories. We asked whether other simple algorithmic changes such as clipping the $T_{i j}$ matrix or a threshold effect produce an equivalent improvement in memory performance. These two do not, presumably because they lack the essential element of the present scheme, that is, the feedback via the algorithm of information about the accessibility of particular states. We believe the results found will be insensitive to whether the state component values are taken as 0 and 1 or \pm 1 .

The REM sleep hypothesis of Crick and Mitchison' refers to higher level processing. Our example illustrates that from a mathematical viewpoint the general idea could work as they described. If the Crick-Mitchison hypothesis is correct, one might ask about correlations between the structure of the spurious linkages in modelling and the strange associations present in dreams.

We thank F. Crick and D. Willshaw for discussions. This work was supported in part by NSF grant DMR-8107494 and by the System Development Foundation.

Received 31 December 1982; accepted 15 May 1983.

. Crick, F. C. \& Mitchison, G. Nature 304, 111-114 (1983).

Hopfield, J. J. Proc. natn. Acad. Sci. U.S.A. 79, 2554-2558 (1982).

\section{A language-specific comprehension strategy}

\section{Anne Cutler*, Jacques Mehlert, Dennis Norris* \& Juan Seguił}

* MRC Applied Psychology Unit, 15 Chaucer Road, Cambridge CB2 2EF, UK

† Laboratoire de Psychologie, CNRS, 54 Boulevard Raspail, 75006 Paris, France

¥ Laboratoire de Psychologie Expérimentale, associé au CNRS, 28 rue Serpente, 75006 Paris, France

Infants acquire whatever language is spoken in the environment into which they are born. The mental capability of the newborn child is not biased in any way towards the acquisition of one human language rather than another. Because psychologists who attempt to model the process of language comprehension are interested in the structure of the human mind, rather than in the properties of individual languages, strategies which they incorporate in their models are presumed to be universal, not language-specific. In other words, strategies of comprehension are presumed to be characteristic of the human language processing system, rather than, say, the French, English, or Igbo language processing systems. We report here, however, on a comprehension strategy which appears to be used by native speakers of French but not by native speakers of English.

Underlying our finding is a.structural difference between the two languages: French and English differ considerably in the degree to which syllable boundaries are clear and unambiguous. In French, syllabic structure is relatively easily determined ${ }^{1}$; 

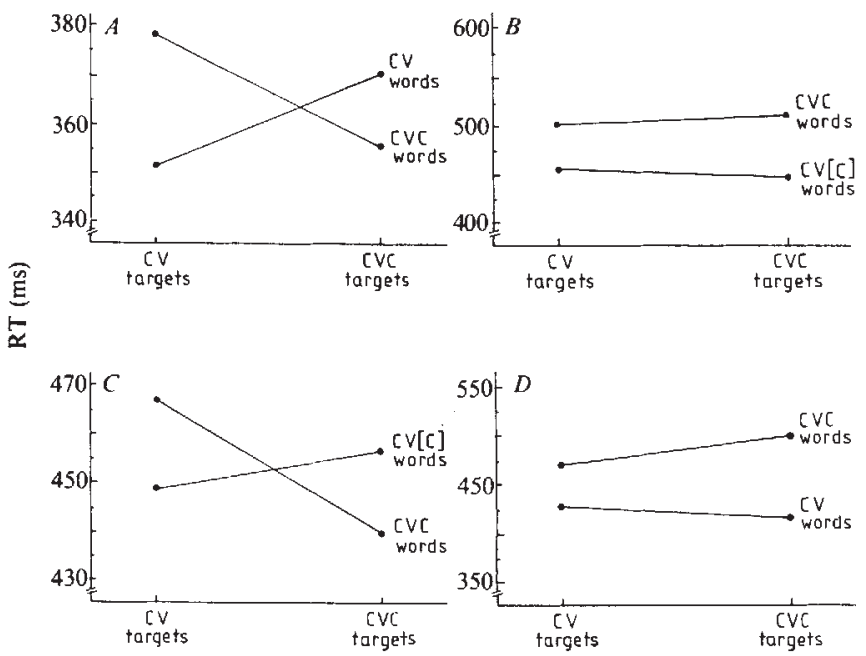

Fig. 1 Mean target detection response time (RT) as a function of size of target sequence (CV, for example, ba- versus CVC, for example, bal-) and size of initial syllable of stimulus word (CV versus CVC for French; CV[C] versus CVC for English), for the following permutations of subjects' native language and stimulus presentation language. $A$, French subjects and words; $B$, English subjects and words; $C$, French subjects, English words; $D$, English subjects, French words.

the first syllable of balance, for example, is clearly $b a$, the first syllable of balcon clearly bal. In English, however, syllable boundaries are often unclear ${ }^{2}$; although English speakers agree that balcony has a syllable boundary after bal, the syllable boundary in balance falls neither after $b a$ nor after bal. Anderson and Jones ${ }^{2}$ would represent the first syllable as [ $\left.\mathrm{bal}\right]$, the second as [lance], the whole word as [ba[1]ance]; the 'l' properly belongs to both the first and the second syllable. Segments which belong to two syllables at once are said to be ambisyllabic; below, we shall use [C] to represent an ambisyllabic consonant.

Previous work ${ }^{3}$, using French words, indicated that in language perception the syllable functions as an effective processing unit; incoming words are processed syllable by syllable. Mehler et $a l^{3}$ asked University of Paris students to listen to lists of unrelated words and to press a response key as fast as possible when they heard a specified word-initial sequence of sounds. This target was either a consonant-vowel (CV) sequence such as $b a$ - or a consonant-vowel-consonant (CVC) sequence such as bal-. The words which began with the sequence had one of two syllabic structures: the initial syllable was either open (CV), as in balance, or closed by a consonant (CVC), as in balcon. They found that response time was significantly faster when the target sequence corresponded exactly to the initial syllable of the word than when the target sequence was equal to more or less of the word than the initial syllable. Thus, the response to the target $b a$ - was faster in the word balance than in balcon, while the target bal-was responded to faster in balcon than in balance (see Fig. $1 A$ ).

This syllabification effect has also been demonstrated in many other studies by Mehler, Segui and colleagues, using a variety of experimental techniques ${ }^{4.5}$. Moreover, sensitivity to the syllable as a unit seems to be natural for pre-linguistic infants ${ }^{6}$, which suggests that it may be a characteristic of the human language processor, independent of particular languages.

Perceptual strategies such as syllabification have presumably been developed because they can speed up the recognition of words. We do not know, however, whether this particular strategy would be as efficient for English as it appears to be for French. Because ambisyllabicity is prevalent in English, and syllable boundaries are therefore hard to identify, it is likely that syllabification would frequently prove difficult, so that it would tend to make the comprehension process less rather than more efficient. Therefore, we replicated the study of Mehler et $a l^{3}$ using English material, and English-speaking subjects.
Twenty-four students at the University of Sussex listened for either CV or else CVC targets in lists of isolated words; the target-bearing words' initial syllables had either CV[C] (for example, balance) or CVC (for example, balcony) structure. The results are presented in Fig. $1 B$; clearly, the response time to $\mathrm{CV}$ and $\mathrm{CVC}$ targets was not significantly different either in CV[C] or in CVC words.

Thus, the structural difference between French and English seems to be reflected in a difference in the way in which the two languages are processed. The syllabification strategy works efficiently in the perception of French, because French is easy to divide into syllables; hence, the syllabification strategy is used by French listeners. English, however, is hard to syllabify, so that such a strategy would be highly inefficient in perception; and indeed, English listeners do not use it. Our results appear to show, therefore, that human listeners use different processing strategies with different languages.

The question then arises of whether such strategies are characteristic of the perceiver (that is, will syllabification be used by French listeners but not by English listeners irrespective of input language?), or whether they are imposed by the characteristics of the speech material (that is, will syllabification be used in the perception of French but not of English irrespective of the perceiver's native language?).

Therefore, we conducted two further experiments in which the subjects' native language and stimulus presentation language differed. Twenty-four University of Sussex students, with only rudimentary knowledge of French, performed the sound sequence detection task on the French material used in the study of Mehler et al. ${ }^{3}$. The results (see Fig. 1D) showed that the performance of the English listeners hearing French material was strikingly similar to that of English listeners who had listened to English material in the previous study. Similarly, when the material from the English-language study was presented to 20 University of Paris V students who were not fluent in English, the performance of these subjects was strikingly similar to the performance of the French listeners who had heard French material in the Mehler et al. study (see Fig. 1C), despite the fact that the language material did not in this case lend itself to the syllabification strategy.

We conclude, therefore, that the syllabification strategy is characteristic of listeners rather than of stimulus language. We suggest that listeners who have acquired French as their native language have developed the syllabification procedure, natural to the human language processing system, into an efficient comprehension strategy. On the other hand, listeners whose native language is English, where this strategy would not necessarily achieve greater comprehension efficiency, have not included syllabification in their repertoire of processing strategies. Further, we suggest that syllabification is only one of a range of possible strategies which the newborn brain has the potential to acquire; whether or not a particular strategy is incorporated in the developing language user's comprehension system will depend on the degree to which the structure of the language being acquired encourages the use of the strategy in question.

This research is a European Psycholinguistics Association collaborative project. We thank P. N. Johnson-Laird and K. E. Patterson for useful comments on the manuscript. J.M. acknowledges support from the Center for Cognitive Science, Massachusetts Institute of Technology, the Harry Frank Guggenheim Foundation, and the Délégation Générale à la Recherche Scientifique et Technique, Décision No. 8070334.

Received 18 April; accepted 17 May 1983.

1. Kaye, J. D. \& Lowenstamm, J. in Phonologie Métrique (Hermann, Paris, 1983).

2. Anderson, J. \& Jones, C. J. Linguistics 10, 1-26 (1974).

3. Mehler, J., Dommergues, J.-Y., Frauenfelder, U. \& Segui, J. J. Verbal Learning Verbal Behav. 20, 298-305 (1981).

4. Segui, J. in Attention and Performance Vol. 10 (Erlbaum, Hillsdale, New Jersey, 1983).

5. Mehler, J. Phil. Trans. R. Soc. B295, 333-352 (1981).

6. Bertoncini, J. \& Mehter, J. Infant Behav. Dev. 4, 247-260 (1981). 